\title{
A SPATIAL CALIBRATION METHOD BASED ON MASTER-SLAVE CAMERA
}

\author{
Hao Shi ${ }^{1}$, Yu Liu, Shiming Lai, Maojun Zhang, Wei Wang
}

Abstract. Recently the Master-Slave camera that composed by fish-eye panoramic camera and PTZ dome camera has been used in many areas. The fish-eye panoramic camera provides 180 degrees field of view (FOV) to monitor the overall situation but the objects are not able to be watched clearly. The PTZ camera can fixate at a target for capturing a high-resolution image. In order to achieve the precise interaction, the pre-processing spatial calibration between these two cameras is required. In this paper, we propose a calibration method to automatically calculate transformation matrix model between the fish-eye coordinate system and the PTZ dome coordinate system by matching the feature points in the scene. The experiment has demonstrated the effectiveness of using this method with high accuracy in short calibration time.

Keywords: Master-Slave camera, Fish-eye Panoramic Camera, PTZ Dome Camera, Spatial Calibration.

\subsection{Introduction}

In recent years, digital video surveillance has already become prevalent in our daily life. Large numbers of monitoring cameras are applied in public and private areas such as government buildings, military bases, schools, car parks, jails etc. The Master-Slave camera that composed by fish-eye panoramic camera and PTZ dome camera has been widely applied in video surveillance field. The fish-eye panoramic camera with a wide field of view acts as the master, which is responsible for acquiring information about the location and general behavior of targets in large scenes. It automatically directs slave cameras to zoom into the targets of interest, which is responsible for observing

\footnotetext{
${ }^{1}$ Hao Shi $(\bowtie)$

College of Information System and Management, National University of Defense Technology

410073 Changsha, China

e-mail: shihao1988@sina.cn

Acknowledgement(s)

This research was partially supported by National Natural Science Foundation (NSFC) of China under project No.61175006, No.61175015, No.61271438, and No.61275016; iCORE (Alberta Innovates) Alberta, and NSERC Canada.
} 
details at a higher resolution. The Master-Slave camera is illustrated in Fig.1.1.

It is one of the important tasks in the application of Master-Slave camera is to control PTZ dome camera to focus on the same target, which is to set the right center of PTZ dome image, detected by the fish-eye panoramic camera. Spatial calibration, can be considered as one of step in the pre-processing process, determines the mapping relationship between each of pixels in fish-eye panoramic image and Pan-Tilt angles of PTZ dome camera. Through this way, the target detected by master camera can be mapped with the slave camera in pixel level.
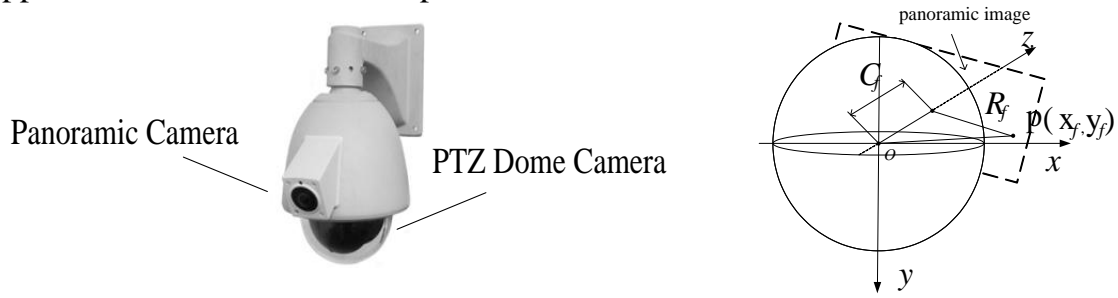

Fig. 1.1 Master-Slave camera

Fig. 1.3 PTZ Image Coordinate System.

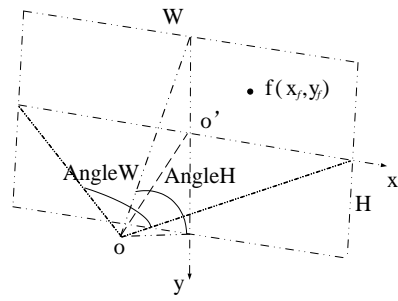

(a)

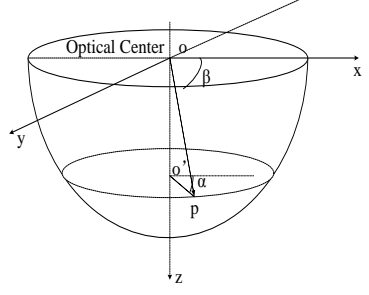

(b)

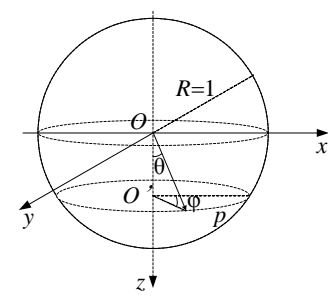

(c)

Fig. 1.2(a) Panoramic Coordinate System. (b) PTZ Coordinate System. (c) Spherical Coordinate System

\subsection{Related Work}

In the literature, several methods have been proposed. Hampapur et al.(1) used two or more calibrated cameras to triangulate a target's position and determine the slave parameters for a third PTZ dome camera that is calibrated to the same coordinate system. Master camera is manually calibrated by designating a number of target areas or regions of interest (such as entrances, high traffic areas etc.) in the master camera's views, all of the areas or regions are focused by the slave camera. Zhou et al.(2) have selected a series of sample master pixel locations in the master camera. Operators 
manually move the slave camera to center the slave image at real-world point of corresponding pixel and record the corresponding rotation angle of PTZ dome camera, then determine non-sample point mapping relationship by a linear interpolation. But linear interpolation is unfit to deformation image leading to many errors. Badri et al.(3) described a method which can automatically sample grids in the master camera image, find proper pan-tilt parameters of slave camera to observe every grid, and extend lookup table by interpolation. Nevertheless, the lookup table is still inaccurate by interpolation for deformation image. Li et al.(4) used a mosaic image created by snapshots of slave camera to estimate the relationship between static master camera plane and pan-tilt controls of slave camera. Through this an efficient and automatic method, the mapping relationship determining by a liner interpolation between pixel in the panoramic image and Pan-Tilt angles of PTZ dome camera is inaccurate.

In this paper, a novel method is proposed to calibrate a Master-Slave camera. The solution is high-precision and time saving. This paper is organized as follows: In section 3, the principle and details of this method are described. In section 4, we show the experimental result. In section 5, we make a conclusion.

\subsection{Calibration}

\subsubsection{Three Coordinate Systems Establishing}

It is the basis of calibration to establish three coordinate systems: a panoramic image coordinate system, a PTZ coordinate system and an auxiliary spherical coordinate system.

Fig.1.2(a) illustrates the panoramic image coordinate system. The $x$ axis and the $y$ axis represent horizontal and vertical direction respectively. The optical center is $o$, and the $o o$ ' is the optical axis. Let $W$ and $H$ be the panoramic image's width and height respectively and let AngleW and AngleH be the panoramic image's horizontal and vertical angle of view respectively. The focal length of fish-eye panoramic camera is denoted as

$$
C_{f}=W / \text { AngleW }=H / \text { AngleH }
$$

$\left(x_{f}, y_{f}\right)$ denotes pixel coordinate in the panoramic image.

As shown in Fig.1.2(b), the PTZ coordinate system contains two parameters. $p$ is a point on the surface of sphere. Let $\alpha$ be the pan angle between $o$ ' $p$ and positive $x$-axis. It increases in the anticlockwise direction viewing from positive $z$-axis, which ranges from $0^{\circ}$ to $359^{\circ}$. Let $\beta$ be the tilt angle which ranges from $0^{\circ}$ to $89^{\circ}$. It is the angle between $o p$ and $X O Y$ plane and increases in the clockwise direction viewing from 
positive $x$-axis. The optical center of PTZ dome camera as the starting point, the section of hemisphere as $X O Y$ plane, the direction downward perpendicular to the $X O Y$ plane as $z$ axis construct the PTZ coordinate system together.

Fig.1.3(c) illustrates the unit spherical coordinate system. Starting point $O$ and every axis correspond to the PTZ dome coordinate system. The coordinate of point $\mathrm{p}$ is denoted as $\left(x_{s}, y_{s}, z_{s}\right) . \varphi$ is the angle between $o$ ' $p$ and positive $x$-axis which increases in the anticlockwise direction viewing from positive $z$-axis and ranges from $0^{\circ}$ to $359^{\circ} . \theta$ is the angle between $o p$ and positive $z$-axis which increases in the anticlockwise direction viewing from positive $x$-axis and ranges from $0^{\circ}$ to $89^{\circ}$.

\subsubsection{Transformation from Panoramic Image Coordinate System to PTZ Coordinate System}

After implementing the transformation from panoramic coordinate system to PTZ coordinate system, we can determine the mapping relationships between each of pixels in fish-eye panoramic image and Pan-Tilt angles of PTZ dome camera. But the transformation process can't be finished in one step directly. We need to use spherical coordinate system to make a linkage. The process is completed in three steps. Assume $\left(x_{f}, y_{f}\right)$ to be any pixel point coordinate in panoramic coordinate system with its corresponding spherical coordinate to be $\left(x_{P_{s}}, y_{P_{s}}, z_{P_{s}}\right)$. Let the mapping point coordinate be $\left(x_{P}, y_{P}, z_{P}\right)$ in PTZ coordinate system and its corresponding spherical coordinate be $\left(x_{H s}, y_{H s}, z_{H s}\right)$. Establishing a mapping $T_{P_{s} \rightarrow H_{s}}$ between $\left(x_{P_{s}}, y_{P_{s}}, z_{P_{s}}\right)$ and $\left(x_{H s}, y_{H_{s}}, z_{H s}\right)$ :

$$
\left(x_{H s}, y_{H s}, z_{H s}\right)=\left(x_{P s}, y_{P s}, z_{P s}\right) \times T_{P s \rightarrow H s}
$$

$T_{P_{s} \rightarrow H s}$ is a $3 * 3$ matrix which denotes the transformation from panoramic spherical coordinate to PTZ dome spherical coordinate. Before the transformation, we need to make sure the two conditions of restriction that our master-slave camera must meet the following requirements:

1. The camera's optical axis is perpendicular to the image plane and point of intersection is at the center of the image plane.

2. The fish-eye panoramic camera's optical center coincides with the PTZ dome camera's optical center.

\subsubsection{Transformation from panoramic coordinate system to spherical coordinate system}

As shown in the Fig.1.3(a), we can obtain the distance between point $p$ and the center: 


$$
R_{f}=\sqrt{x_{f}^{2}+y_{f}^{2}}
$$

Based on the fish-eye imaging model, the radical angle of point $p$ off center is:

$$
\theta=\frac{R_{f}}{C_{f}}
$$

According to $R_{f}$ and $\theta$, the $z$ axis of point $p$ can be calculate. So the transformation is:

$$
\begin{gathered}
x_{P s}=S x_{f} \\
y_{P s}=S y_{f} \\
z_{P s}=S \frac{\sqrt{x_{f}^{2}+y_{f}^{2}}}{\tan \left(\frac{\sqrt{x_{f}^{2}+y_{f}^{2}}}{c_{f}}\right)}
\end{gathered}
$$

$S$ is the normalization constant to make $\sqrt{{x_{P s}{ }^{2}+{y_{P s}}^{2}+z_{P s}{ }^{2}}^{2}}=1$.

\subsubsection{Transformation from PTZ coordinate system to spherical coordinate} system

As PTZ dome camera model is a hemisphere model, the PTZ coordinate system is identical with the spherical coordinate system essentially. The transformation is:

$$
\begin{gathered}
x_{H s}=\cos \alpha \cos \beta=\cos \varphi \sin \theta \\
y_{H s}=\sin \alpha \cos \beta=\sin \varphi \sin \theta \\
z_{H s}=\sin \beta=\cos \theta
\end{gathered}
$$

According to the transformation by two steps as described above, the final transformation from panoramic spherical coordinate system to PTZ dome spherical coordinate system can be obtained:

$$
\left(x_{H s}, y_{H s}, z_{H s}\right)=\left(x_{P s}, y_{P s}, z_{P s}\right) \times T_{P s \rightarrow H s}
$$

\subsubsection{Feature Point Matching and Matrix Solution}

In our experiment, the mapping $T_{P_{s} \rightarrow H_{s}}$ is calculated through a process of selecting sampling points. In order to solve the $3 \times 3$ matrix $T_{P_{S} \rightarrow H s}$, we select $\mathrm{n}(\mathrm{n} \geqslant 3)$ PTZ 
dome image, each of which provides a set of points. This procedure requires a method of detecting and matching visual feature robust to scale, rotation, view-point, and lightning. The Scale Invariant Feature Transform (SIFT)(5)exhibits great performance under these constraints. So we employ SIFT method to detect feature point in both panoramic image and PTZ dome image. Panoramic image and PTZ dome image are two input parameters in SIFT method. Let $\left\{P_{H i}^{j}\right\}=\left\{\left(\mathrm{x}_{H i}^{j}, \mathrm{y}_{H i}^{j}, \mathrm{z}_{H i}^{j}\right)\right\}^{T},(i=1, \cdots n, n \geqslant 3$; $j=1, \cdots m, m>1)$ be the $j$ th feature point's spherical coordinate of the $i$ th PTZ dome image. Let $\left\{P_{P i}^{j}\right\}=\left\{\left(\mathrm{x}_{P i}^{j}, \mathrm{y}_{P i}^{j}, \mathrm{z}_{P i}^{j}\right)\right\}^{T},(i=1, \cdots n, n \geqslant 3 ; j=1, \cdots m, m>1)$ be the panoramic spherical coordinate that corresponds to $\left\{P_{H i}^{j}\right\}$. Let $A_{H i}$ be feature point matrix in the $i$ th PTZ dome image and $A_{P i}$ be matching feature point matrix in the panoramic image. $T_{i}$ is transformation matrix between the $i$ th PTZ dome image and panoramic image and we solve for it using least square method.

$$
\begin{gathered}
A_{H i}=\left[P_{H i}{ }^{1}, P_{H i}{ }^{2}, \cdots, P_{H i}{ }^{m}\right] \\
A_{P i}=\left[P_{P i}{ }^{1}, P_{P i}{ }^{2}, \cdots, P_{P i}{ }^{m}\right] \\
T_{i}=A_{P i} \times\left(A_{H i}{ }^{T} A_{H i}\right)^{-1} A_{H i}{ }^{T}
\end{gathered}
$$

Let $P_{H i}{ }^{0}$ be the center point spherical coordinate of the $i$ th PTZ dome image and $P_{P i}{ }^{0}$ be the panoramic spherical coordinate that corresponds to $P_{H i}{ }^{0}$.

$$
P_{P i}^{0}=T_{i} \times P_{H i}^{0} \quad(i=1, \cdots n)
$$

Let $A_{H}$ be center point spherical coordinate matrix in the PTZ dome image and $A_{P}$ be matching point matrix in the panoramic image.

$$
\begin{gathered}
A_{H}=\left[P_{H 1}{ }^{0}, P_{H 2}{ }^{0}, \cdots, P_{H n}{ }^{0}\right] \\
A_{P}=\left[P_{P 1}{ }^{0}, P_{P 2}{ }^{0}, \cdots, P_{P n}{ }^{0}\right] \\
T_{P s \rightarrow H s}=A_{P} \times\left(A_{H}{ }^{T} A_{H}\right)^{-1} A_{H}
\end{gathered}
$$

The aim of the whole calibration process is to solve for the matrix $\mathrm{T}_{\mathrm{Ps}_{s} \rightarrow \mathrm{Hs}}$. When given any pixel $p\left(x_{f}, y_{f}\right)$, we can obtain corresponding rotation angle $(\alpha, \beta)$.

\subsection{Experiments and Results}

The proposed method has been tested by computer program. Fig.1.4(a) shows the monitoring results of master-slave camera cooperates together. The top one is panoramic image and we have selected four areas which have the distinguished feature. The other images below are PTZ dome images with zooming. Fig.1.4(b) shows the calibration results and the best linear fit. As can be seen, the real pan angles match well 
with the requested angles from $0^{\circ}$ to $360^{\circ}$ and there is no obvious error. Similar good results are obtained for tilt angles (not shown here). Fig.1.4(c) shows the mean error between requested pixel $\left(p_{x}, p_{y}\right)$ and real pixel $\left(p_{x}{ }^{\prime}, p_{y}{ }^{\prime}\right)$ to analyze the distribution of error. We select 170 sampling points from panoramic image to analyze errors. The error calculation formula is :

$$
\text { centering_error }=\sqrt{\left(p_{x}-p_{x}^{\prime}\right)^{2}+\left(p_{y}-p_{y}^{\prime}\right)^{2}}
$$

The dark blue area represents 0 35 pixels, the green area represents 35 70 pixels and the red area represents 70 105 pixels. As the PTZ dome image size used is 1920 * 1080 pixels and its horizontal coverage is 55 , we can find that about 35 pixels are equivalent to $1^{\circ}$. As can be seen, error can be controlled within $1^{\circ}$ over $95 \%$ of the area.

In the calibration process, there are three main factors to influence the accuracy of calibration. The first factor is PTZ dome camera we have used can't accurate to $0.1^{\circ}$. If so, 3.5 pixels will be equivalent to $0.1^{\circ}$. Centering error can reduce greatly. The second factor is selection of the PTZ dome images' position. In our experiment we have selected 6 PTZ dome image. If the 6 positions are completely uniform distribution in the panoramic image, the red area can't appear in Fig.1.4(c). The third factor is feature point matching, the number and accuracy of measurement of match points influence the result of calibration directly. In order to show the accuracy we mark all pairs of matched points, which display with different colors. If too many errors are displayed, PTZ dome camera will capture another image.

The method's another advantage is time savings. The core process contains subtracting images and image matching. Subtracting one image takes about 2 seconds. For one pair of images, image matching takes about 5 seconds. So the whole process (6 PTZ dome images) takes less than 1 minute.

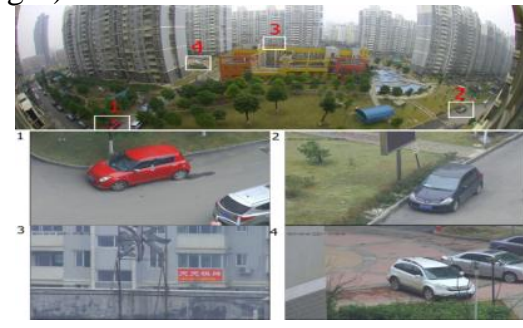

(a)

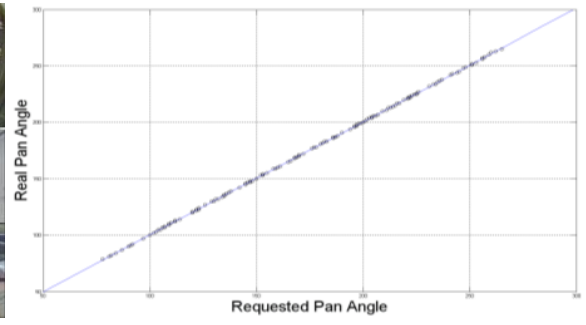

(b) 


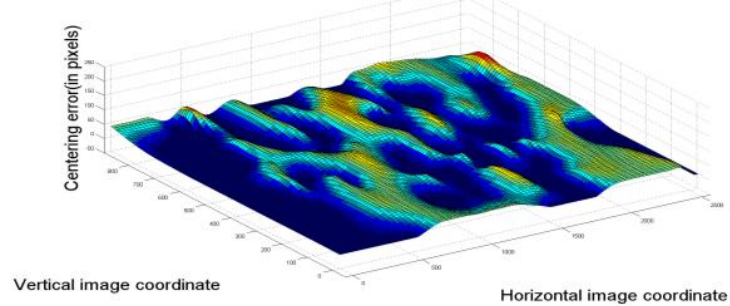

(c)

Fig. 1.4(a) Monitoring results of master-slave camera. (b) Relation between requested and real pan angle. (c) Mean error between requested and real pixel

\subsection{Conclusions}

In this paper, we have presented a novel method for calibrating the master-slave camera. We obtain the mapping relationships between master camera and slave camera by getting a transformation matrix model between their coordinate system. In this process, SIFT algorithm is adopted to match feature points. The availability and accuracy of the method is validated by the experiments shown in this paper.

\subsection{References}

1. Hampapur A, Pankanti S, Senior A, Tian Y-L, Brown L, Bolle R, editors. Face cataloger: Multiscale imaging for relating identity to location. Proceedings IEEE Conference on Advanced Video and Signal Based Surveillance, 2003; 2003: IEEE.

2. Zhou X, Collins RT, Kanade T, Metes P, editors. A master-slave system to acquire biometric imagery of humans at distance. First ACM SIGMM international workshop on Video surveillance; 2003: ACM.

3. Badri J, Tilmant C, Lavest J-M, Pham Q-C, Sayd P. Camera-to-camera mapping for hybrid pan-tiltzoom sensors calibration. Image Analysis: Springer; 2007. p. 132-41.

4. You L, Li S, Jia W, editors. Automatic Weak Calibration of Master-Slave Surveillance System Based on Mosaic Image. Pattern Recognition (ICPR), 2010 20th International Conference on; 2010: IEEE.

5. Lowe DG. Distinctive image features from scale-invariant keypoints. International journal of computer vision. 2004;60(2):91-110. 